\section{Skeletal Radiology turns 40}

\author{
Murali Sundaram • Daniel I. Rosenthal • Juerg Hodler
}

(C) ISS 2011

At the conclusion of this year, Skeletal Radiology, the journal of the International Skeletal Society, will have published the final issue of its 40th volume. The journal (and the ISS itself) was created to help bring order to the world of musculoskeletal diagnosis. The five decades of its existence has recorded tremendous progress. The first issue of the journal was published a couple of years after the first computed tomographic (CT) head scanners became functional in London and Manchester (United Kingdom) The advent of magnetic resonance imaging (MRI), advances in ultrasound, and continued advances in CT and PACS, as well as progress in histochemistry, genetics, and chemotherapy have changed diagnostic and therapeutic musculoskeletal practice in ways that simply could not have been imagined at the time of publication of the first volume of Skeletal Radiology.

Radiology itself has largely evolved from a field of generalists, and a second career choice, into a subspecialized and highly sought-after career. Musculoskeletal radiology is now a recognized subspecialty in which most practitioners have had fellowship training. Pathology has likewise become increasingly specialized as techniques other than light microscopy have come to dominate clinical practice. Orthopedic oncology has acquired ever more powerful tools

M. Sundaram $(\bowtie)$

Cleveland Clinic, Lerner College of Medicine,

Cleveland $\mathrm{OH}$, USA

e-mail: sundarm@ccf.org

D. I. Rosenthal

Massachusetts General Hospital, Harvard University,

Boston MA, USA

J. Hodler

University Hospital Zurich,

Zurich, Switzerland to treat diseases; chemotherapy, special alloys, implants, biological substances, and computer-guided surgeries are some examples.

We have chosen to devote this issue exclusively to review papers that encompass the many advances in knowledge and clinical practice over the past five decades.

Just as clinical practice has changed, so has Skeletal Radiology, which has grown from a single volume of 4 issues and 220 pages to one of 12 issues and 1,200 pages. In 2003, the journal launched an electronic submission and review system. Within 12 months of the launch, 500 manuscripts had been uploaded. In its fourth year of on-line existence, 2,500 manuscripts were uploaded.

Some things have not changed. Unlike most journals, Skeletal Radiology has chosen to devote a section of its pages to case reports because of the uniqueness of these reports, which also constantly highlight the interdisciplinary nature of clinical practice.

The reviews in this issue are necessarily limited in scope. However, it seemed appropriate to attempt a brief summation of what has changed as we pause briefly to look both backward and simultaneously forward. We hope that you will enjoy the scope and spectrum of topics covered in this issue as much as we have enjoyed its compilation. 